\title{
A NOTE ON RESTRICTION THEOREMS FOR SEMISTABLE SHEAVES
}

\author{
ADRIAN LANGER
}

\begin{abstract}
We prove a new restriction theorem for semistable sheaves on varieties in all characteristics strengthening previous results. We also prove restriction theorem for strong semistability for varieties with some non-negativity constrains on the cotangent bundle (e.g., most of Fano and CalabiYau varieties).
\end{abstract}

\section{Introduction}

The main result of this note is a generalization of the following theorem from the characteristic zero case to positive characteristic.

THEOREM 1.1. Let $X$ be a smooth projective variety of dimension $n$ defined over an algebraically closed field $k$ of characteristic 0 . Let $H$ be a very ample divisor on $X$ and let $E$ be an $\mathrm{H}$-semistable torsion free sheaf of rank $r \geq 2$ on X. Let us take an integer a such that

$$
\left(\begin{array}{c}
a+n \\
n
\end{array}\right)>\frac{1}{2}\left(\max \left\{\frac{r^{2}-1}{4}, 1\right\} H^{n}+1\right) \Delta(E) H^{n-2}+1 .
$$

Then for a general divisor $D \in|a H|$ the restriction $E_{D}$ is $H_{D}$-semistable.

To avoid introducing a complicated notation we defer a precise formulation of the generalization of Theorem 1.1 to Section 2 (see Theorem 3.1). The above stated theorem is also new although a slightly weaker result can be obtained by combining Flenner's restriction theorem and the author's version of Bogomolov's restriction theorem (see [6, Theorem 5.2]). In positive characteristic the analogue of Theorem 1.1 (i.e., Theorem 3.1) is new even in the well-known and very much studied case of rank 2 vector bundles on $\mathbb{P}^{2}$.

Obviously, the theorem in positive characteristic implies the characteristic zero version by usual arguments involving reduction modulo large characteristic. Hence in the following we consider only the positive characteristic version. In positive characteristic $p$, the theorem improves earlier bounds on degree of hypersurfaces for which the restriction is semistable from roughly the discriminant to the $n$-th root of discriminant. In particular, it shows that for every strongly semistable sheaf $E$ (of rank $r<p+2$ ) on a variety of dimension at least 2 there exists a computable integer $a_{0}$ such that for all integers $a>a_{0}$ the restriction of $\left(F^{m}\right)^{*} E$ is semistable on the generic hypersurface of degree $a p^{m}$. This generalizes the main result of [9] (see [9, Theorem 2.7]), where a similar theorem was proven for some special classes of varieties. In fact, for varieties considered in [9] (and many more) we prove that there exists a restriction theorem for strong semistability (see Theorem 4.1).

The proof of our theorem is a combination of proofs of the Grauert-Mülich, Flenner's and Bogomolov's restriction theorems. The main new features are a non-separable descent and

Received by the editors May 28, 2009. Revision received April 3. 2010. 
the use of author's version of Bogomolov's inequality in positive characteristic (see Theorem 2.1) to elementary transformations along non-reduced divisors.

The paper is organized as follows. In Section 1 we recall a few basic results. In Section 2 we prove the main result: a new restriction theorem for semistability. In Section 3 we prove a restriction theorem for strong semistability on varieties with certain non-negativity constrains on the cotangent bundle (this class includes, e.g., non-supersingular K3 surfaces and most of Fano and Calabi-Yau varieties).

1.1. Notation and conventions. Let $S$ be a scheme defined over an algebraically closed field $k$ of characteristic $p$. Then by $F_{S}^{r}: S \rightarrow S$ we denote the $r$-th absolute Frobenius morphism of $S$ (i.e., the $p^{r}$-th power mapping on $\mathscr{O}_{S}$ ).

Let $X$ be an $S$-scheme and let $X_{S}^{(r)}$ denote the fiber product of $X$ and $S$ over the $r$-th Frobenius morphism $F_{S}^{r}: S \rightarrow S$. The $r$-th absolute Frobenius morphism of $X$ induces the $r$-th relative Frobenius morphism $F_{X / S}^{r}: X \rightarrow X_{S}^{(r)}$. This morphism decomposes into a composition of $r$ relative Frobenius morphisms $F_{X / S}^{r}=F_{X_{S}^{(r-1)} / S} \ldots F_{X_{S}^{(1)} / S} F_{X / S}$. For a morphism $\pi: X \rightarrow S$ the induced morphism $X_{S}^{(r)} \rightarrow S$ is denoted by $\pi_{S}^{(r)}$. The above notation is best illustrated by the following diagram:

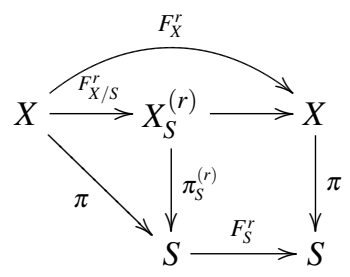

When $S=$ Spec $k$ then to simplify notation we denote $X_{S}^{(r)}$ by $X^{(r)}$. As we will need a few different Frobenius morphisms we will use a slightly inconsistent notation and for a morphism $\pi: X \rightarrow S, \pi^{(r)}$ will denote not the morphism $\pi_{k}^{(r)}$ but the morphism $X^{(r)} \rightarrow S^{(r)}$ induced by $\pi$.

Semistability in this paper will always mean slope semistability with respect to the considered ample divisor.

\section{Preliminaries}

In this section we gather a few auxiliary results.

Let $(X, H)$ be a smooth polarized variety of dimension $n$ defined over an algebraically closed field $k$ of characteristic $p$. Let $E$ be a rank $r$ torsion free sheaf on $X$. Then one can define the slope of $E$ by $\mu(E)=c_{1} E \cdot H^{n-1} / r$. The slope of a maximal destabilizing subsheaf of $E$ is denoted by $\mu_{\max }(E)$ and that of minimal destabilizing quotient by $\mu_{\min }(E)$. The discriminant of $E$ is defined by $\Delta(E)=2 r c_{2}(E)-(r-1) c_{1}^{2}(E)$.

Let us recall that in positive characteristic the degree of instability of Frobenius pull backs of $E$ is measured by the following (well-defined and finite) number:

$$
L_{\max }(E)=\lim _{k \rightarrow \infty} \frac{\mu_{\max }\left(\left(F^{k}\right)^{*} E\right)}{p^{k}} .
$$


Let us set

$$
L_{X}= \begin{cases}\frac{L_{\max }\left(\Omega_{X}\right)}{p} & \text { if } \mu_{\max }\left(\Omega_{X}\right)>0, \\ 0 & \text { if } \mu_{\max }\left(\Omega_{X}\right) \leq 0 .\end{cases}
$$

The following theorem is a slight strengthening of [6, Theorem 5.1]. It can be proved in the same way except that at the end of proof one needs to use [7, Theorem 2.17] instead of [6, Theorem 3.3].

THEOREM 2.1. For an arbitrary torsion free sheaf $E$ we have the following inequality:

$$
H^{n} \cdot \Delta(E) H^{n-2}++r^{2}\left(\mu_{\max }(E)-\mu(E)\right)\left(\mu(E)-\mu_{\min }(E)\right)+\frac{r^{2}(r-1)^{2}}{4} L_{X}^{2} \geq 0 .
$$

We will also need a corollary to the following theorem of Ilangovan, Mehta and Parameswaran:

THEOREM 2.2. (see [4, Theorem 3.1]) Let $G$ be a connected reductive algebraic group over $k$ and let $G \rightarrow S L(V)$ be a low height representation (see $[4,2.1]$ for the definition of low height representations). Then for every semistable principal $G$-bundle $E$ on $X$, the induced vector bundle $E(V)$ is also semistable with respect to the same polarization.

COROLLARY 2.3. If $E_{1}$ and $E_{2}$ are semistable sheaves and $p>\operatorname{rk} E_{1}+\operatorname{rk} E_{2}-2$ then $E_{1} \otimes E_{2}$ is semistable.

Finally, let us recall the following result which in characteristic zero was proven by $\mathrm{H}$. Flenner (see [2, Corollary 2.2]) and in arbitrary characteristic it was (almost) proven by $\mathrm{H}$. Brenner (see [1, Corollary 7.1]):

THEOREM 2.4. The kernel $K_{a}$ of the evaluation map $H^{0}\left(\mathscr{O}_{\mathbb{P}}(a)\right) \otimes \mathscr{O}_{\mathbb{P} n} \rightarrow \mathscr{O}_{\mathbb{P}^{n}}(a)$ is semistable.

In fact, Brenner in his paper uses the characteristic zero assumption when using Klyachko's results (see [1, remark on p. 416, before Lemma 6.1]). However, it was remarked by M. Perling in [10] that Klyachko's results are valid in an arbitrary characteristic. In fact, Perling's description is better suited to generalizing [1, Lemma 6.1] as it uses toric sheaves rather than just toric vector bundles. A weaker form of the above theorem, but still sufficient for our applications, is the main result of Trivedi's paper [12]. In particular, Trivedi proves that

$$
\mu_{\max }\left(K_{a}^{*}\right) \leq \frac{a}{\left(\begin{array}{c}
\left.\frac{a}{2}\right\rceil+n-1 \\
n-1
\end{array}\right)} .
$$

\section{New restriction theorem for semistability}

THEOREM 3.1. Let $k$ be an algebraically closed field of characteristic $p>0$. Let $X$ be a smooth $n$-dimensional $(n \geq 2) k$-variety with a very ample divisor $H$. Let $E$ be an $H$ semistable torsion free sheaf of rank $r \geq 2$ on $X$. Let us assume that $p \geq r-1$ and let us take an integer a such that

$$
\left(\begin{array}{c}
a+n \\
n
\end{array}\right)>\frac{1}{2}\left(\max \left\{\frac{r^{2}-1}{4}, 1\right\} H^{n}+1\right)\left(\Delta(E) H^{n-2}+\frac{r^{2}(r-1)^{2} L_{X}{ }^{2}}{4 H^{n}}\right)+1 .
$$

Then for a general divisor $D \in|a H|$ the restriction $E_{D}$ is $H_{D}$-semistable. 
Proof. Let us assume that the restriction of $E$ to a general divisor in $|a H|$ is not $H$-semistable.

Let $\Pi$ denote the complete linear system $|a H|$. Let $Z=\{(D, x) \in \Pi \times X: x \in D\}$ be the incidence variety with projections $p: Z \rightarrow \Pi$ and $q: Z \rightarrow X$. Let $Z_{t}$ denote the scheme theoretic fibre of $p$ over $t \in \Pi$. Let $0 \subset E_{0} \subset E_{1} \subset \cdots \subset E_{l}=q^{*} E$ be the relative HarderNarasimhan filtration of $q^{*} E$ with respect to $p$ (see [3, Theorem 2.3.2 and Remark 2.3.3]). By definition there exists a nonempty open subset $U$ of $\Pi$ such that all quotients $E^{i}=E_{i} / E_{i-1}$ are flat over $U$ and such that for every $t \in U$ the fibres $\left(E_{\bullet}\right)_{t}$ form the Harder-Narasimhan filtration of $E_{t}=\left(q^{*} E\right)_{Z_{t}}$ (we can also assume that all fibers $Z_{t}$ for $t \in U$ are smooth).

Now the proof splits into several steps.

Step 1. In this step we find an upper bound on a certain difference of slopes of consecutive quotients in the Harder-Narasimhan filtration of restriction of $E$ to a general divisor in $|a H|$ (see inequality (1)). This is done using an inseparable descent.

Assume that the filtration $E_{\bullet}$ descends under the $s$-th relative Frobenius morphism $F_{Z / X}^{s}$ : $Z \rightarrow Z_{X}^{s}$ but it does not descend under the $(s+1)$-th relative Frobenius morphism $F_{Z / X}^{s+1}: Z \rightarrow$ $Z_{X}^{(s+1)}$. To simplify notation let us denote $Z_{X}^{(s)}$ by $Z^{\prime}$ and $q_{X}^{(s)}$ by $q^{\prime}$. Let $E_{\bullet}^{\prime}$ be a filtration of $\left(q^{\prime}\right)^{*} E$ such that $E_{\bullet} \simeq\left(F_{Z / X}^{(s)}\right)^{*} E_{\bullet}^{\prime}$. Since

$$
\left(q^{\prime}\right)^{*} E=\left(F_{Z^{\prime} / X}\right)^{*}\left(\left(q_{X}^{(s+1)}\right)^{*} E\right)
$$

there exists a canonical relative connection

$$
\nabla_{\text {can }}:\left(q^{\prime}\right)^{*} E \rightarrow \Omega_{Z^{\prime} / X} \otimes\left(q^{\prime}\right)^{*} E .
$$

By our assumption and Cartier's theorem (see, e.g., [5, Theorem 5.1]) there exists some index $i_{0}$ such that $E_{i_{0}}^{\prime}$ is not preserved by $\nabla_{\text {can }}$. Then the connection induces a non-zero $\mathscr{O}_{Z^{\prime}}$-homomorphism

$$
E_{i_{0}}^{\prime} \rightarrow \Omega_{Z^{\prime} / X} \otimes\left(\left(q^{\prime}\right)^{*} E\right) / E_{i_{0}}^{\prime}
$$

After pulling-back to $Z$ we get a non-zero $\mathscr{O}_{Z}$-homomorphism

$$
E_{i_{0}} \rightarrow\left(F_{Z / X}^{s}\right)^{*} \Omega_{Z^{\prime} / X} \otimes\left(q^{*} E\right) / E_{i_{0}} .
$$

After restricting to a general fibre $Z_{t}$ of $p$ we see that

$$
\mu_{\min }\left(\left(E_{i_{0}} \otimes\left(q^{*} E / E_{i_{0}}\right)^{*}\right)_{Z_{t}}\right) \leq \mu_{\max }\left(\left(\left(F_{Z / X}^{s}\right)^{*} \Omega_{Z^{\prime} / X}\right)_{Z_{t}}\right) .
$$

By assumption, quotients $E_{Z_{t}}^{i}$ are semistable for a general point $t \in \Pi$. As the sum of ranks of $E^{i}$ and $E^{j}$ is less than or equal to $r \leq p+1$, the Ilangovan-Mehta-Parameswaran theorem (see Corollary 2.3) implies semistability of sheaves $E_{Z_{t}}^{i} \otimes\left(E_{Z_{t}}^{j}\right)^{*}$ for all $i, j$. Let us set $r_{i}=\operatorname{rk}\left(E_{Z_{t}}^{i}\right)$ and $\mu_{i}=\mu\left(E_{Z_{t}}^{i}\right)$. Then by the above we have

$$
\mu_{i_{0}}-\mu_{i_{0}+1} \leq \mu_{\max }\left(\left(\left(F_{Z / X}^{s}\right)^{*} \Omega_{Z^{\prime} / X}\right)_{Z_{t}}\right) .
$$

Step 2. In this step we introduce some notation and we recall a lower bound on differences $\mu_{i}-\mu_{i+1}$ (see inequality (2)).

Let $\mathscr{K}$ be the kernel of the evaluation map $H^{0}\left(X, \mathscr{O}_{X}(a H)\right) \otimes \mathscr{O}_{X} \rightarrow \mathscr{O}_{X}(a H)$. Then $Z=$ $\mathbb{P}_{X}\left(\mathscr{K}^{\vee}\right)$ and $q$ corresponds to the natural projection $\mathbb{P}_{X}\left(\mathscr{K}^{\vee}\right) \rightarrow X$. It is easy to see that $Z^{\prime}=\mathbb{P}_{X}\left(\left(F^{s}\right)^{*} \mathscr{K}^{\vee}\right)$ and $\left(F_{Z / X}^{s}\right)^{*} \mathscr{O}_{\mathbb{P}_{X}\left(\left(F^{s}\right)^{*} \mathscr{K}^{\vee}\right)}(1)=\mathscr{O}_{\mathbb{P}_{X}\left(\mathscr{K}^{\vee}\right)}\left(p^{s}\right)$. Let us note that the Picard group of $\mathbb{P}_{X}\left(\left(F^{s}\right)^{*} \mathscr{K}^{\vee}\right)$ is generated over $\left(q^{(s)}\right)^{*} \operatorname{Pic} X$ by $\mathscr{O}_{\mathbb{P}\left(\left(F^{s}\right)^{*} \mathscr{K}^{\vee}\right)}(1)$. Therefore we can write $\operatorname{det}\left(E^{i}\right)^{\prime} \simeq \mathscr{O}_{Z^{\prime}}\left(-b_{i}^{\prime}\right) \otimes\left(q^{(s)}\right)^{*} \mathscr{L}_{i}$ for some integers $b_{i}^{\prime}$ and line bundles $\mathscr{L}_{i}$ on $X$. 
Then $\operatorname{det} E^{i} \simeq \mathscr{O}_{Z}\left(-p^{s} b_{i}^{\prime}\right) \otimes q^{*} \mathscr{L}_{i}$. We know that $\mu_{i}>\mu_{i+1}$ and $\mu_{i}=\frac{\operatorname{deg} E_{Z_{t}}^{i}}{r_{i}}$. By the above $\operatorname{deg} E_{Z_{t}}^{i}=\operatorname{deg}\left(\left.\mathscr{L}_{i}\right|_{Z_{s}}\right)=a L_{i} H^{n-1}$, where $L_{i}=c_{1} \mathscr{L}_{i}$. Hence

$$
\mu_{i}-\mu_{i+1}=\frac{a L_{i} H^{n-1}}{r_{i}}-\frac{a L_{i+1} H^{n-1}}{r_{i+1}} \geq \frac{a}{\max \left\{\frac{r^{2}-1}{4}, 1\right\}} .
$$

Step 3. In this step we find a precise bound on $\mu_{\max }\left(\left(\left(F_{Z / X}^{s}\right)^{*} \Omega_{Z^{\prime} / X}\right) Z_{t}\right)$. This step can be summed up in the following lemma, whose proof is similar to proof of Flenner's restriction theorem (see [2, Proposition 1.10]; we will use [3, Theorem 7.1.1] as reference as it will be easier to follow the proof):

LEMMA 3.2. If $a p^{s}<\left(\begin{array}{c}a+n \\ n\end{array}\right)-1$ then

$$
\mu_{\max }\left(\left(\left(F_{Z / X}^{s}\right)^{*} \Omega_{Z^{\prime} / X}\right)_{Z_{t}}\right) \leq \frac{a^{2} p^{s} H^{n}}{\left(\begin{array}{c}
a+n \\
n
\end{array}\right)-a p^{s}-1} .
$$

Proof. First we need to reduce to the case $X=\mathbb{P}^{n}$. This can be done in the same way as Steps 2 and 3 in proof of [3, Theorem 7.1.1] and therefore we skip it. Let us set $N=\left(\begin{array}{c}a+n \\ n\end{array}\right)$ and let $K$ be the kernel of the evaluation map $H^{0}\left(\mathscr{O}_{\mathbb{P}^{n}}(a)\right) \otimes \mathscr{O}_{\mathbb{P}^{n}} \rightarrow \mathscr{O}_{\mathbb{P}^{n}}(a)$. As in Step 4 of proof of [3, Theorem 7.1.1], using the Veronese embedding $\mathbb{P}^{n} \hookrightarrow \mathbb{P}^{N-1}$ one can see that we only need to show that

$$
\mu_{\max }\left(\left(\left(F^{s}\right)^{*} K^{*}\right)_{D}\right) \leq \frac{a^{2} p^{s}}{N-a p^{s}-1},
$$

where $D$ is a general degree $a$ hypersurface in $\mathbb{P}^{n}$. Now let us recall that $K$ is semistable (see Theorem 2.4). Since every semistable bundle on $\mathbb{P}^{n}$ is strongly semistable (as $\mu_{\max }\left(\Omega_{\mathbb{P}^{n}}\right)<0$ ) we see that $\left(F^{s}\right)^{*} K^{*}$ is also semistable. Moreover, by the Ramanan-Ramanathan theorem (see [11, Theorem 3.23]) the bundles $\Lambda^{q}\left(\left(F^{s}\right)^{*} K^{*}\right)$ are also semistable for all $q$. Hence

$$
H^{0}\left(\mathbb{P}^{n}, \bigwedge^{q}\left(\left(F^{s}\right)^{*} K^{*}\right) \otimes \mathscr{O}_{\mathbb{P}^{n}}(b)\right)=0
$$

if $0>\mu\left(\bigwedge^{q}\left(\left(F^{s}\right)^{*} K^{*}\right) \otimes \mathscr{O}_{\mathbb{P}^{n}}(b)\right)=b-q p^{s} \mu(K)$, which is equivalent to $b<-\frac{q a p^{s}}{N-1}$. Now we can come back to Step 4 of proof of [3, Theorem 7.1.1] and finish in the same way.

Step 4. Gathering information obtained in previous steps we find in this step a lower bound on $a p^{s}$ (see inequality (3)), where $s$ is the same as in Step 1.

Lemma from Step 3, together with inequalities (1) and (2) imply that if $a p^{s}<\left(\begin{array}{c}a+n \\ n\end{array}\right)-1$ then

This inequality is equivalent to

$$
\frac{a}{\max \left\{\frac{r^{2}-1}{4}, 1\right\}} \leq \frac{a^{2} p^{s} H^{n}}{\left(\begin{array}{c}
a+n \\
n
\end{array}\right)-a p^{s}-1} .
$$

$$
a p^{s} \geq \frac{1}{H^{n} \max \left\{\frac{r^{2}-1}{4}, 1\right\}+1}\left(\left(\begin{array}{c}
a+n \\
n
\end{array}\right)-1\right) .
$$

Obviously, the above inequality is also satisfied if $a p^{s} \geq\left(\begin{array}{c}a+n \\ n\end{array}\right)-1$.

Step 5. This step containing the rest of the proof is quite lengthy. It is devoted to finding an upper bound on $a p^{s}$. First we prove that the restrictions of $E$ to some non-reduced divisors are unstable. Then the strategy is quite similar to the author's proof of Bogomolov type restriction 
theorem [6, Theorem 5.2]. Namely, we can perform elementary transformations with respect to these non-reduced divisors and use Theorem 2.1 to find an upper bound on degree of divisors for which the restriction is unstable. One of the new features is that to get interesting inequalities, instability of restrictions to non-reduced divisors needs to be understood for locally free subsheaves rather than for all pure subsheaves.

Let us consider the commutative diagram

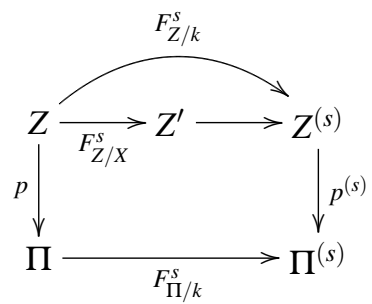

Let $p^{\prime}$ be the composition of $Z^{\prime} \rightarrow Z^{(s)}$ and $Z^{(s)} \rightarrow \Pi^{(s)}$. The fibers of this map are nonreduced divisors of the form $p D$ for $D \in \Pi$.

Let us recall that the sheaves $E^{i}$ are locally free on an open subset whose intersection with each fiber $Z_{t}, t \in U$, contains all points in codimension 1 in $Z_{t}$ (this follows from the fact that $E_{Z_{t}}^{i}$ are torsion free and $E^{i}$ is locally free at the points where $E_{Z_{t}}^{i}$ is locally free; see, e.g., [3, Lemma 2.1.7]).

Let us note that if $X \rightarrow Y$ is a faithfully flat morphism of schemes and for some coherent $\mathscr{O}_{Y}$-module $G$ its pull-back $f^{*} G$ is locally free then $G$ is locally free. This can be easily seen locally using, e.g., the fact that projective modules over local rings are free. This also implies that if $f^{*} G$ is torsion free then $G$ is also torsion free.

Let us set $U^{\prime}=F_{\Pi / k}^{s}(U)$. Since $\left(F_{Z / X}^{s}\right)^{*}\left(E^{\prime}\right)^{i}=E^{i}$ this fact implies that $\left(E^{\prime}\right)^{i}$ are locally free in codimension 1 on fibers $Z_{t}^{\prime}$ of $p^{\prime}$ for $t \in U^{\prime}$. Using [3, Lemma 2.1.4] one can in fact see that all factors $\left(E^{\prime}\right)^{i}$ are flat over $U^{\prime}$. In particular, for $t \in U^{\prime}$ the fibres $\left(E_{\bullet}^{\prime}\right)_{t}$ form a filtration of $E_{t}^{\prime}=\left(\left(q^{\prime}\right)^{*} E\right)_{Z_{t}^{\prime}}$ with quotients that are pure $\mathscr{O}_{Z_{t}^{\prime}}$-modules. These quotients are also locally free in codimension 1 (note that this does not follow from the purity as the fibers $Z_{t}$ are non-reduced).

Let us take $t \in U$ and let $t^{\prime}$ be its image in $U^{\prime}$. Let $D$ denote the image of $Z_{t}$ under $p$. Then $Z_{t^{\prime}}^{\prime}$ is isomorphic to the non-reduced divisor $p^{s} D$. Let $S$ and $T$ denote the sheaves on $X$ corresponding to $\left(E_{l-1}^{\prime}\right)_{Z_{t^{\prime}}^{\prime}}$ and $\left(\left(E^{\prime}\right)^{l}\right)_{Z_{t^{\prime}}^{\prime}}$. Let $G$ be the kernel of the composition $E \rightarrow$ $E_{p^{s} D} \rightarrow T$. Then we have two short exact sequences:

$$
0 \rightarrow G \rightarrow E \rightarrow T \rightarrow 0
$$

and

$$
0 \rightarrow E\left(-p^{s} D\right) \rightarrow G \rightarrow S \rightarrow 0
$$

We can use the first sequence to compute $\Delta(G)$ :

$$
\Delta(G)=\Delta(E)-\rho(r-\rho) p^{2 s} D^{2}+2\left(r c_{1}(T)-(r-\rho) p^{s} D c_{1}(E)\right),
$$

where $\rho$ is the rank of $S$ on $n D$ (which is the same as the rank of $S_{D}$ on $D$ ). Now we need the following lemma:

LEMMA 3.3. Let $D \subset X$ be a smooth divisor on a smooth projective variety $X$ of dimension at least 2 and let $d \geq 1$ be an integer. Let $F$ be a coherent $\mathscr{O}_{d D}$-module that is locally free at each codimension 1 point of $D$. Then $c_{1}(F)=d \cdot c_{1}\left(F_{D}\right)$ (as Chern classes of sheaves on $X$ ). 
Proof. Taking hyperplane sections we can easily reduce the assertion to the surface case. Let us note that for every $i \leq d$ we have the following short exact sequences of $\mathscr{O}_{d D}$-modules

$$
0 \rightarrow \mathscr{O}_{(i-1) D}(-D) \rightarrow \mathscr{O}_{i D} \rightarrow \mathscr{O}_{D} \rightarrow 0 .
$$

Since $F$ is a locally free $\mathscr{O}_{d D}$-module, the corresponding sequences remain exact after tensoring with $F$. Therefore we have a filtration $0=F_{0} \subset F_{1} \subset \cdots \subset F_{d-1} \subset F_{d}=F$ of $F$ with sheaves $F_{j}=F \otimes \mathscr{O}_{j D}(-(d-j) D)$ and quotients $F_{D}(-(p-j) D)$. This easily implies the required equality of Chern classes.

By the above lemma we have

$$
\Delta(G) H^{n-2}=\Delta(E) H^{n-2}-\rho(r-\rho) p^{2 s} D^{2} H^{n-2}+2 p^{s}\left(r c_{1}\left(T_{D}\right)-(r-\rho) D c_{1}(E)\right) H^{n-2} .
$$

From the definition of the filtration $E_{\bullet}$ we know that

$$
\left(r c_{1}\left(T_{D}\right)-(r-\rho) D c_{1}(E)\right) H^{n-2}=\rho \operatorname{deg}\left(E_{Z_{t}}^{l}\right)-(r-\rho) \operatorname{deg}\left(\left(E_{l-1}\right)_{Z_{t}}\right)<0 .
$$

But we already proved that both $\operatorname{deg}\left(E_{Z_{t}}^{l}\right)$ and $\operatorname{deg}\left(\left(E_{l-1}\right)_{Z_{t}}\right)$ are divisible by $a$ so we get

$$
\Delta(G) H^{n-2} \leq \Delta(E) H^{n-2}-\rho(r-\rho) p^{2 s} D^{2} H^{n-2}-2 a p^{s} .
$$

Using semistability of $E$ and $E\left(-p^{s} D\right)$ we get

$$
\mu_{\max }(G)-\mu(G)=\mu_{\max }(G)-\mu(E)+\frac{r-\rho}{r} p^{s} D H^{n-1} \leq \frac{r-\rho}{r} a p^{s} H^{n}
$$

and

$$
\mu(G)-\mu_{\min }(G)=\mu\left(E\left(-p^{s} D\right)\right)-\mu_{\min }(G)+\frac{\rho}{r} p^{s} D H^{n-1} \leq \frac{\rho}{r} a p^{s} H^{n} .
$$

Hence, applying Theorem 2.1 to $G$, we obtain

$$
\begin{aligned}
-\frac{r^{2}(r-1)^{2}}{4} L_{X}{ }^{2} \leq & H^{n} \cdot \Delta(G) H^{n-2}+r^{2}\left(\mu_{\max }(G)-\mu(G)\right)\left(\mu(G)-\mu_{\min }(G)\right) \\
\leq & H^{n} \cdot \Delta(E) H^{n-2}-\rho(r-\rho) a^{2} p^{2 s}\left(H^{n}\right)^{2}-2 a p^{s} H^{n} \\
& +r^{2}\left(\frac{r-\rho}{r} a p^{s} H^{n}\right)\left(\frac{\rho}{r} a p^{s} H^{n}\right) .
\end{aligned}
$$

Therefore we get

$$
2 a p^{s} \leq \Delta(E) H^{n-2}+\frac{r^{2}(r-1)^{2} L_{X}^{2}}{4 H^{n}},
$$

which together with inequality (3) gives a contradiction to our assumptions on $a$.

Example 3.4. For the projective plane $L_{\mathbb{P}^{2}}=0$. In particular, if for $E$ in Theorem 3.1 we take the tangent bundle, then the theorem implies that the restriction of $T_{\mathbb{P}^{2}}$ to a general curve of degree $\geq 2$ is semistable. Obviously, this result is the best possible as the restriction of $T_{\mathbb{P}^{2}}$ to any line is not semistable.

It is easy to see that the technique of proof of Step 5 of Theorem 3.1 gives the following restriction theorem: 
THEOREM 3.5. Let E be an $H$-semistable torsion free sheaf and let a be an integer such that

$$
a>\frac{1}{2} \Delta(E) H^{n-2}+\frac{r^{2}(r-1)^{2} L_{X}^{2}}{8 H^{n}} .
$$

Then for a general divisor $D \in|a H|$ the restriction $E_{D}$ is $H_{D}$-semistable.

Note that the above theorem is valid in arbitrary characteristic. In particular, to get the characteristic zero statement we replace in Step 5 all Frobenius morphisms by identities (so we consider only reduced divisors) and following the proof we arrive at inequality $2 a \leq$ $\Delta(E) H^{n-2}$.

This improves the bound given in [6, Corollary 5.4] although it works only for a general divisor in $|a H|$. This theorem, together with Flenner's theorem implies Theorem 1.1. As was noted in Introduction, Theorem 1.1 follows also from Theorem 3.1 by reduction modulo large characteristic. More precisely, let $R \subset k$ be a finitely generated $\mathbb{Z}$-algebra such that there exists a projective morphism $f: \mathscr{X} \rightarrow \operatorname{Spec} R$ which after base change to $k$ gives $X$. Taking appropriate localizations we can assume that $f$ is smooth. Then there exists a non-negative integer $a$ such that $T_{\mathscr{X} / R} \otimes \mathscr{O}_{\mathscr{X} / R}(a)$ is $f$-globally generated. This shows that $L_{\max }\left(\Omega_{\mathscr{X}_{t}}\right)$ can be bounded from the above by the same constant for all fibers $\mathscr{X}_{t}$ of $f$. Hence $L_{\mathscr{X}_{t}}$ tends to 0 when the characteristic of the residue field $k(t)$ goes to infinity. Now by openness of semistability Theorem 3.1 implies Theorem 1.1.

\section{Restriction theorem for strong semistability}

Below we sketch a proof of a restriction theorem for strong semistability for a very general member of sufficiently ample linear system on some varieties. The proof is a modification of the proof of [7, Theorem 2.20].

THEOREM 4.1. Let $X$ be a smooth $n$-dimensional $(n \geq 2)$ variety with a very ample divisor H. Let us assume that $\mu_{\max }\left(\Omega_{X}\right) \leq 0$. Let $E$ be an $H$-semistable torsion free sheaf of rank $r \geq 2$ on $X$. Let us take an integer a such that

$$
a>\frac{1}{2} \max \left\{\Delta(E) H^{n-2}, \Delta\left(\Omega_{X}\right) H^{n-2}+n(n-1)^{2} \frac{\left(K_{X} H^{n-1}\right)^{2}}{H^{n}}\right\} .
$$

and

$$
\frac{\left(\begin{array}{c}
a+n \\
n
\end{array}\right)-1}{a}>\max \left\{\frac{r^{2}-1}{4}, 1\right\} H^{n}+1 .
$$

Then the restriction of $E$ to the generic hypersurface in $|a H|$ is strongly H-semistable.

Proof. By assumption $\mu_{\max }\left(\Omega_{X}\right) \leq 0$, so $E$ is strongly $H$-semistable and $L_{X}=0$. By Theorem 3.5 and our assumption on $a$ we also know that $E_{D}$ is semistable for a general divisor $D \in$ $|a H|$.

As in the proof of Theorem 3.1 let $\Pi$ denote the complete linear system $|a H|$ and let $Z=$ $\{(D, x) \in \Pi \times X: x \in D\}$ be the incidence variety with projections $p: Z \rightarrow \Pi$ and $q: Z \rightarrow X$. Let $\eta \in \Pi$ be the generic point of $\Pi$ (i.e., a non-closed point whose closure is $\Pi$ ). We need to prove that $E_{Z_{\eta}}$ is strongly semistable. If it is not then there exists $m$ such that the relative Harder-Narasimhan filtration $0 \subset E_{0} \subset E_{1} \subset \cdots \subset E_{l}=q^{*}\left(\left(F^{m}\right)^{*} E\right)$ of $\left(F^{m}\right)^{*} E$ with respect to $p$ is non-trivial and the quotients of the Harder-Narasimhan filtration of the restriction of $E_{Z_{\eta}}$ are strongly semistable. Let us take such minimal $m$. 
If the filtration $E_{\bullet}$ descends under the geometric Frobenius morphism $Z \rightarrow Z^{(1)}$ then the descended filtration destabilizes $\left(F^{m-1}\right)^{*} E$ and the quotients of the Harder-Narasimhan filtration of the restriction of $E_{Z_{\eta}}$ are strongly semistable, which contradicts our choice of $m$. So the filtration $E_{\bullet}$ does not descend. Now the canonical connection $\nabla_{\text {can }}$ on $F^{*}\left(\left(F^{m-1}\right)^{*} E\right)$ induces a non-zero $\mathscr{O}_{X}$-homomorphism

$$
E_{i} \rightarrow \Omega_{Z} \otimes\left(F^{m}\right)^{*} E / E_{i} .
$$

This implies that

$$
\mu_{\min }\left(\left(E_{i} \otimes\left(\left(F^{m}\right)^{*} E / E_{i}\right)^{*}\right)_{Z_{s}}\right) \leq \max \left(\mu_{\max }\left(\left(\Omega_{Z / X}\right)_{Z_{s}}\right), \mu_{\max }\left(\left(q^{*} \Omega_{X}\right)_{Z_{s}}\right)\right)
$$

for a general fibre $Z_{s}$ of $p$. Now we claim that

$$
\mu_{\max }\left(\left(q^{*} \Omega_{X}\right)_{Z_{s}}\right) \leq 0
$$

This follows from $\mu_{\max }\left(\Omega_{X}\right) \leq 0$ and the equality

$$
\mu_{\max }\left(\left(\Omega_{X}\right)_{D}\right)=a \mu_{\max }\left(\Omega_{X}\right)
$$

for a general divisor $D \in|a H|$. To prove this last equality let us consider the HarderNarasimhan filtration $0=G_{0} \subset G_{1} \subset \cdots \subset G_{m}=\Omega_{X}$ of the cotangent bundle. Set $G^{i}=$ $G_{i} / G_{i-1}, n_{i}=\operatorname{rk} F_{i}$ and $v_{i}=\mu\left(F_{i}\right)$. Since $G^{i}$ are semistable we have $\Delta\left(G^{i}\right) H^{n-2} \geq 0$ by Theorem 2.1. By the Hodge index theorem and [6, Lemma 1.4] we have

$$
\begin{aligned}
\frac{\Delta\left(\Omega_{X}\right) H^{n-2}}{n} & =\sum \frac{\Delta\left(G^{i}\right) H^{n-2}}{n_{i}}-\frac{1}{n} \sum_{i<j} n_{i} n_{j}\left(\frac{c_{1} G^{i}}{n_{i}}-\frac{c_{1} G^{j}}{n_{j}}\right)^{2} H^{n-2} \\
& \geq \sum \frac{\Delta\left(G^{i}\right) H^{n-2}}{n_{i}}-\frac{1}{n H^{n}} \sum_{i<j} n_{i} n_{j}\left(v_{i}-v_{j}\right)^{2} \\
& \geq \frac{\Delta\left(G^{l}\right) H^{n-2}}{n_{l}}+\frac{n}{H^{n}}\left(\mu_{\max }\left(\Omega_{X}\right)-\mu\left(\Omega_{X}\right)\right)\left(\mu_{\min }\left(\Omega_{X}\right)-\mu\left(\Omega_{X}\right)\right)
\end{aligned}
$$

for every integer $l$. Therefore

$$
\begin{aligned}
\frac{\Delta\left(G^{l}\right) H^{n-2}}{n_{l}} & \leq \frac{\Delta\left(\Omega_{X}\right) H^{n-2}}{n}+\frac{n}{H^{n}}\left(\mu_{\max }\left(\Omega_{X}\right)-\mu\left(\Omega_{X}\right)\right)\left(\mu\left(\Omega_{X}\right)-\mu_{\min }\left(\Omega_{X}\right)\right) \\
& \leq \frac{\Delta\left(\Omega_{X}\right) H^{n-2}}{n}+\frac{n(n-1)}{H^{n}}\left(\mu\left(\Omega_{X}\right)\right)^{2}
\end{aligned}
$$

and hence Theorem 3.5 implies that $\left(G^{l}\right)_{D}$ is semistable for a general divisor $D \in|a H|$, which proves our claim.

Now the rest of proof is as before in proof of Theorem 3.1: by the Ramanan-Ramanathan theorem we have

$$
\mu_{i}-\mu_{i+1} \leq \mu_{\max }\left(\left(\Omega_{Z / X}\right)_{Z_{s}}\right)
$$

and we know that

$$
\frac{a}{\max \left\{\frac{r^{2}-1}{4}, 1\right\}} \leq \mu_{i}-\mu_{i+1}
$$

and

$$
\mu_{\max }\left(\left(\Omega_{Z / X}\right)_{Z_{s}}\right) \leq \frac{a^{2} H^{n}}{\left(\begin{array}{c}
a+n \\
a
\end{array}\right)-a-1} .
$$

This gives a contradiction with our assumptions on $a$. 
One can easily generalize Theorem 4.1 to general complete intersections in varieties with $\mu_{\max }\left(\Omega_{X}\right) \leq 0$ but explicit bounds become harder to write down. One can also see that instead of $\mu_{\max }\left(\Omega_{X}\right) \leq 0$ it is sufficient to assume that $\mu_{\max }\left(\Omega_{X}\right) \leq \frac{1}{\max \left\{\frac{r^{2}-1}{4}, 1\right\}}$.

Condition $\mu_{\max }\left(\Omega_{X}\right) \leq 0$ is satisfied in many cases when $K_{X}$ is not ample. For example, it is satisfied for abelian varieties, varieties of separated flags and for smooth toric varieties. Since in characteristic zero the tangent bundle of a Calabi-Yau variety (i.e., with $K_{X}=0$ ) is semistable (for all polarizations), the reduction of such a variety modulo a general prime also satisfies assumption of our theorem. More precisely, one can prove that non-uniruled Calabi-Yau varieties satisfy condition $\mu_{\max }\left(\Omega_{X}\right) \leq 0$ (see [8]).

\section{Acknowledgements}

The author would like to thank Vikram Mehta and Vijaylaxmi Trivedi for useful conversations. The author was partially supported by a Polish KBN grant (contract number NN201265333).

\section{References}

[1] H. Brenner, Looking out for stable syzygy bundles. With an appendix by Georg Hein, Adv. Math. 219 (2008), 401-427.

[2] H. Flenner, Restrictions of semistable bundles on projective varieties, Comment. Math. Helv. 59 (1984), 635-650.

[3] D. Huybrechts, M. Lehn, The geometry of moduli spaces of sheaves, Aspects of Mathematics 31, 1997.

[4] S. Ilangovan, V. B. Mehta, A. J. Parameswaran, Semistability and semisimplicity in representations of low height in positive characteristic, in A tribute to C. S. Seshadri (Chennai, 2002), 271-282, Trends Math., Birkhäuser, Basel, 2003.

[5] N. Katz, Nilpotent connections and the monodromy theorem: Applications of a result of Turrittin, Publ. Math. IHES 39 (1970), 175-232.

[6] A. Langer, Semistable sheaves in positive characteristic, Ann. of Math. 159 (2004), 251-276.

[7] A. Langer, Moduli spaces of sheaves and principal $G$-bundles, in Algebraic Geometry: Seattle 2005, eds. D. Abramovich et al., Proc. Symp. Pure Math. 80 (2009), 273-308.

[8] A. Langer, A note on foliations in positive characteristic, in preparation.

[9] V. B. Mehta, V. Trivedi, On some Frobenius restriction theorems for semistable sheaves, Proc. Indian Acad. Sci. Math. Sci. 119 (2009), 109-118.

[10] M. Perling, Graded rings and equivariant sheaves on toric varieties, Math. Nachr. 263/264 (2004), 181-197.

[11] S. Ramanan, A. Ramanathan, Some remarks on the instability flag, Tohoku Math. J. 36 (1984), 269-291.

[12] V. Trivedi, Semistablity of syzygy bundles on projective spaces in positive characteristics, preprint arXiv:0804.0547.

1. Institute of Mathematics, Warsaw University, Ul. Banacha 2, 02-097 WARSZAWA, Poland, 2. Institute of Mathematics, Polish ACAdemy of SCIENCES, Ul. ŚNiAdeckich 8, 00-956 WarszaWA, POLAND

E-mail address: alan@mimuw.edu.pl 